\title{
Correlation of adverse effects of cisplatin administration in patients affected by solid tumours: A retrospective evaluation
}

\author{
LAURA ASTOLFI ${ }^{1}$, SARA GHISELLI ${ }^{2}$, VALERIA GUARAN ${ }^{1}$, MILVIA CHICCA $^{3}$, \\ EDI SIMONI $^{1}$, ELENA OLIVETTO ${ }^{1}$, GIORGIO LELLI ${ }^{4}$ and ALESSANDRO MARTINI ${ }^{2}$ \\ ${ }^{1}$ Bioacoustics Research Laboratory, ${ }^{2}$ ENT Surgery, Department of Neurosciences, University of Padua, I-35129 Padua; \\ ${ }^{3}$ Department of Life Sciences and Biotechnology, University of Ferrara, I-44121 Ferrara; \\ ${ }^{4}$ Clinical Oncology Unit, St. Anna University-Hospital, I-44121 Ferrara, Italy
}

Received November 8, 2012; Accepted December 5, 2012

DOI: $10.3892 /$ or.2013.2279

\begin{abstract}
Cisplatin is the most common antineoplastic drug used for the therapy of solid tumours. To date, researchers have focused on the dosage to be administered for each specific tumour, mainly considering the local adverse effects. The aim of this study was to correlate the severity of the adverse effects with: i) the dosage of cisplatin; ii) the specific site of the tumour; iii) the association with other drugs; and iv) the symptoms. We analysed data from 123 patients with 11 different tumour classes undergoing therapy from 2007 to 2008 at St. Anna Hospital (Ferrara, Italy), using the Spearman non-parametric correlation index. Even though significant correlations were found among the variables, the overall results showed that the main factor influencing the severity of the adverse effects was the dosage of cisplatin administered.
\end{abstract}

\section{Introduction}

The majority of therapies for malignant tumours are based on chemotherapeutic drugs with cytotoxic effects, which cause death of tumour cells by direct damage to DNA or by inhibition of cell division. Unfortunately, these drugs are mostly unspecific, therefore, their administration often causes extended tissue toxicity (1).

Cisplatin, or cis-diamminedichloroplatinum(II) (CDDP) was the first platinum-based anticancer drug developed for clinical purposes. It is employed in children for treatment of haematological tumours and in adults for treatment of solid tumours such as testicular $(2)$, prostatic and ovarian $(3,4)$, uterine, cervix, breast, bladder, stomach $(5,6)$ colon, brain,

Correspondence to: Dr Laura Astolfi, Bioacoustics Research Laboratory, Department of Neurosciences, University of Padua, Via G. Orus 2b, I-35129 Padua, Italy

E-mail: laura.astolfi@unipd.it

Key words: adverse effect correlation, cisplatin toxicity, solid tumours, chemotherapy head-neck $(7,8)$ and both non-small and small-cell lung cancer $(9,10)$.

The intravenous administration of cisplatin causes an increase in initial tissue accumulation and in plasma levels for an extended time. Cisplatin levels are high in plasma as the drug reversibly binds to $>90 \%$ of proteins; traces of the drug are often detected years after chemotherapy $(11,12)$. Brouwers and co-researchers (13), during a 6-year follow-up screening, demonstrated that plasma levels of cisplatin have a half life $(t / 2)$ of 28.5 months $(14,15)$. Moreover, the elimination of the drug is not the same in all tissues; elimination is faster in more rapidly regenerating tissues in comparison to slower ones and each tissue has its own t/2 (16). The plasma levels of cisplatin depend upon several factors: cumulative dose, follow-up time, age of patient, glomerular filtration speed (GFR) during chemotherapy, use of sodium thiosulphate (STS) during cisplatin chemotherapy and method of administration $(11,13)$. A higher initial dose of the drug causes a higher tissue concentration and a longer time for excretion, yet STS binding to cisplatin lowers its initial tissue accumulation (16).

Cisplatin has both biliary $(\sim 10 \%)$ and urinary $(90 \%)$ clearance. The urinary clearance of cisplatin is characterised by an initial fast excretion phase $(20 \mathrm{~min})$, followed by a second slower phase (60-70 min) and a third very slow and incomplete phase $(24 \mathrm{~h})(17,18)$.

The activity of cisplatin and the appearance of the side effects depend on pharmacological parameters such as the dosage (single and cumulative) and administration (schedule and means), but also on systemic and individual conditions such as skin pigmentation, age, diet, blood $\mathrm{pH}$ and interactions with radiotherapy (19-21). Certain effects are dose-dependent; thus, they can be controlled but not prevented (22-24).

Among the adverse effects that may develop, the most frequent ones are gastrointestinal symptoms. More than $90 \%$ of patients experience nausea and vomiting; these symptoms are counteracted by the administration of antiemetic drugs such as the antagonist of serotonin receptor $3\left(5-\mathrm{HT}_{3}\right)$ and dexamethasone. In a smaller number of cases, general symptoms are detected, such as fever, hyposthenia, altered sleep-wake cycle, myelosuppression and alteration in the liver, skin and respiratory apparatus. Among the negative side effects with a 
more or less severe involvement of tissues include neurotoxicity, nephrotoxicity and ototoxicity $(2,13,25-27)$.

The different responses to cisplatin treatment depend on individual factors and on resistance mechanisms. Resistance mechanisms include: reduction in intracellular drug accumulation, drug inactivation by the cytosol, changes in DNA repair mechanisms and alterations in proteins involved in apoptosis $(18,28-31)$. Resistance may be induced by only one or several of the above mentioned mechanisms (31). The reduction in intracellular accumulation may depend upon an increased drug outflow or a reduced inflow through the cell membrane. The inflow by membrane channels may be modified by several compounds including amphotericin B, aldehydes, inhibitors of $\mathrm{Na}-\mathrm{K}$ adenosine triphosphatase and ouabain (18). Molecules such as glutathione and metallothioneins may react with cisplatin at the intracellular level, inactivating it and preventing its binding to DNA. Others may reduce its chemotherapeutic efficacy by modifying the DNA repair mechanisms (32); among these there are the DNA polymerase inhibitors (zidovudine and ganciclovir), the inhibitors of topoisomerase II (etoposide and novobiocin), the methylxanthines (caffeine and pentoxifylline) and specific chemotherapeutic drugs (5-fluorouracil, cytarabine and hydroxyurea) $(33,34)$.

The different compounds studied for interference with cisplatin toxicity inlcude sodium thiosulphate, D-methionine, vitamins C and E, Gingko biloba extract and dexamethasone $(25,35-38)$.

To date, most clinical literature data report that the efficacy and side effects of cisplatin treatment are associated with other compounds in patients belonging to homogeneous groups or with tumours in specific body sites. The purpose of the present research was to evaluate the incidence of side effects in patients with different types of tumours, undergoing chemotherapy with cisplatin. Records of the patients retrospectively examined were heterogeneous, in order to verify i) whether various chemotherapy combinations increase the sensitivity of the organism to the toxic effects of the drug; ii) whether a direct correlation could be detected between the tumour site and a specific side effect; and finally iii) whether the side effects were reciprocally related. For this purpose, we examined the medical records of 123 patients treated with cisplatin in the same hospital (St. Anna University Hospital, Ferrara, Italy) during 2007 and 2008, with special attention to the dosages and side effects reported.

\section{Materials and methods}

Study population. The medical records of 123 patients (81 males and 42 females), undergoing chemotherapy during 2007 and 2008 at the Clinical Oncology Unit, St. Anna University Hospital in Ferrara (Italy), were retrospectively examined in agreement with Italian privacy and sensitive data laws (D.Lgs 196/03) and according to the institutional guidelines of the St. Anna University Hospital.

Tumour distribution. All malignant tumours were classified according to the Italian Association of Cancer Registries (AIRTUM, Associazione Italiana Registri Tumori) and the International Classification of Diseases. The cancers were recognised as follows: lung, head and neck, gynaecological, melanoma, thymoma, gastric, occult, neuroendocrine, urothelial, hepatic and thyroid.

Treatment. Doses and methods of cisplatin treatment were modulated according to the drug therapeutic plan (alone, in association with other chemotherapeutic agents or with radiotherapy), depending on the tumour type and on the conditions of the patients.

Cisplatin (cis-diamminedichloroplatinum(II), CDDP) was administered alone or with gemcitabine (GEM), epirubicin (EPI), etoposide (VP-16), 5-fluorouracil (5-FU), dacarbazine (DTIC), vinorelbine (VNR) or in a combination called EDOC (EPI + CDDP + vincristine + cyclophosphamide). In all cases, the drug treatment was preceded by hydration and by antiemetic treatment with dexamethasone and serotonin (5-hydroxytriptamine 3,5- $\mathrm{HT}_{3}$ ) (from $30 \mathrm{~min}$ to $1 \mathrm{~h}$ and $30 \mathrm{~min}$ before chemotherapy). The pretreatment was recommended by the American Society of Clinical Oncology (ASCO, 2006) since cisplatin is one of the chemotherapeutic agents with the most severe emetic side effects (incidence $>90 \%$ ). Although the daily standard dose of dexamethasone is $20 \mathrm{mg}$, in most cases the prescribed daily dose was $8 \mathrm{mg}$ in $100 \mathrm{ml}$ of saline solution, administered intravenously. The $5-\mathrm{HT}_{3}$ drugs are a group of antagonists of the $5-\mathrm{HT}_{3}$ serotonin receptor (ondansetron, granisetron or dolasetron). The method of administration (oral or intravenous) does not influence their efficacy in controlling symptoms. The administration of the chemotherapeutic drugs was also preceded by administration of two diuretics (furosemide and mannitol). The hydration of the patients undergoing chemotherapy with cisplatin is necessary to reduce dehydration and the relevant nephrotoxic effects of the drug. When cisplatin is administered with GEM, 5-FU or VNR, additional administration of dexamethasone is required (a total of $16 \mathrm{mg}$ ), and when the therapy follows the EDOC scheme, the amount of serotonin is doubled as well. Among the 123 patients studied, 63 were also treated by radiotherapy, particularly when affected by head-neck tumours.

Classification of adverse effects. The side effects observed, following the Common Terminology Criteria for Adverse Events (CTCAE) v3.0 (National Cancer Institute, 2006) were respectively categorised as follows: auditory/ear (ototoxicity), blood/bone marrow (haematological toxicity), constitutional symptoms, dermatology/skin (dermatological disorders), gastrointestinal (gastrointestinal disorders), hepatobiliary/pancreas (hepatic toxicity), neurology (neurotoxicity), pulmonary/upper respiratory (respiratory disorders), renal/genitourinary (nephrotoxicity) and sexual/reproductive function (genital apparatus disorders). Changes in sleep-wake cycle were classified in a separate category, termed sleep-wake disorders, as they are not clearly categorised by CTCAE.

Statistical analysis. The collected data represent cancer prevalence in 2008 among oncological patients of the St. Anna University Hospital of Ferrara, undergoing therapy for a maximum of 6 years.

For all data, the average values and standard deviations were calculated for dosages and the frequency of side effects detected in all patients and for patients grouped by tumour 
Table I. Patient characteristics.

\begin{tabular}{|c|c|c|c|c|c|c|c|c|c|c|c|c|c|}
\hline \multirow[b]{2}{*}{ Gender } & \multirow{2}{*}{$\begin{array}{l}\text { Years since } \\
\text { diagnosis }\end{array}$} & \multirow[b]{2}{*}{ Age $\pm S D$} & \multicolumn{11}{|c|}{ Tumour classes } \\
\hline & & & $\mathrm{L}$ & $\mathrm{HN}$ & GN & M & $\mathrm{TM}$ & GS & $\mathrm{O}$ & $\mathrm{N}$ & $\mathrm{U}$ & $\mathrm{HE}$ & TY \\
\hline \multicolumn{14}{|l|}{ Male } \\
\hline & 1 & $61 \pm 9.9$ & 14 & 7 & - & 1 & - & 2 & 1 & 1 & 1 & - & - \\
\hline & 2 & $62 \pm 8.2$ & 23 & 9 & - & 1 & 2 & 1 & - & - & 1 & - & - \\
\hline & 3 & $60 \pm 9.2$ & 6 & 5 & - & 1 & - & - & - & 1 & - & 1 & - \\
\hline & 4 & $66 \pm 13.4$ & 2 & - & - & - & - & - & - & - & - & - & - \\
\hline & 6 & 39 & - & 1 & - & - & - & - & - & - & - & - & - \\
\hline \multicolumn{14}{|l|}{ Female } \\
\hline & 1 & $56 \pm 11.5$ & 1 & 1 & 5 & 1 & - & - & - & 1 & - & - & - \\
\hline & 2 & $60 \pm 13.4$ & 3 & 4 & 5 & 1 & - & - & 1 & - & - & - & 2 \\
\hline & 3 & $58 \pm 8.3$ & 4 & 1 & 7 & - & 1 & - & - & - & 1 & 1 & - \\
\hline & 4 & $53 \pm 5.7$ & 1 & - & 1 & - & - & - & - & - & - & - & - \\
\hline
\end{tabular}

Number and mean age ( \pm standard deviation) (in years) of oncological patients grouped according to gender, years since diagnosis and tumour class. L, lung; HN, head and neck; GN, gynaecological; M, melanoma; TM, thymoma; GS, gastric; O, occult; N, neuroendocrine; U, urothelial; HE, hepatic; TY, thyroid.

type. Concerning the possible association between the examined variables, we calculated the Spearman non-parametric correlation. The data were verified by r-Pearson and were plotted in dendrograms by the unweighted pair-group method using arithmetic averages. Statistica 7 (StatSoft srl., Italy, 2005) software was used.

\section{Results}

Patient characteristics. The patients had a mean age of $60.0 \pm 9.9$ (SD) years (calculated when they received the first treatment with cisplatin) and an age range of 35-81 years. An overview of the data is shown in Table I, which reports the number of patients by gender, years since diagnosis and type of cancer.

Concerning the incidence of tumours in relation to gender, we calculated the relative percentage for each tumour typology. Thyroid and obviously gynaecological tumours were restricted to females, while gastric carcinoma was detected only in males. A frequency of 50-60\% was detected in occult carcinoma, hepatic carcinoma and melanoma (one male patient had hepatic carcinoma and one female had biliary tract cancer). In all other tumours examined the incidence was higher in males in comparison to females.

As shown in Table I, the tumours with the highest prevalence were those of the lung and head and neck, with a 4:1 male:female ratio. The third most frequent tumours in women were gynaecological tumours. In all other cases, the number of affected individuals ranged from 2 (occult, hepatic and thyroid cancers) to a maximum of 5 (melanoma). In Table I different tumour histotypes were grouped together. For example, all female tumours ( 5 cases of cervical cancer, 4 of ovarian cancer, 4 of breast cancer, 3 of endometrial cancer, one of breast-endometrial cancer and one of vulvar cancer) were grouped under the GN (gynaecological) class. Lung cancers [42 non-small cell lung cancer (NSCLC), 9 small cell lung cancer (SCLC), one sarcomatoid carcinoma and two unspecified cases] were grouped under the L (lung) class.

Recording of adverse effects. The majority of patients had gastrointestinal disorders, such as nausea and vomiting, diarrhoea, constipation, epigastralgia, pyrosis, dysphasia, postprandial abdominal bloating sensation, white tongue, dysgeusia and taste impairment. Among the other adverse effects, constitutional symptoms included hyposthenia and asthenia, fever, weight loss and appetite loss. Cisplatin myelosuppression caused haematological toxic effects, such as anaemia, leukopenia, neutropenia and thrombocytopenia. The dermatological disorders included alopecia, itchiness, skin rash, edema, arm phlebitis and mucositis. Neurotoxicity mainly involved the peripheral system in comparison to the central nervous system. The most common symptoms were paraesthesia, followed by cephalalgia, speech impairments, aphasia, agnosia, lipothymia (near-fainting syndrome), convulsions, panic and transient ischemic attacks (mini-strokes), visual failure, sensory-motor deficits and motor coordination impairments. Nephrotoxicity included electrolyte alterations

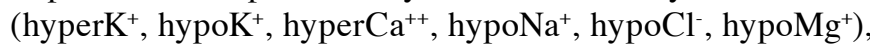
an increase in blood nitrogen, creatinine and urea, pollakiuria (abnormally frequent urination), hematuria, oliguria, polyuria, urinary tract infections, and kidney spasms to renal insufficiency. Hepatic toxicity was characterised by hepatomegaly and a rise in hepatic enzymes (transaminases, bilirubin, $\gamma$-glutamyl transpeptidases). Respiratory disorders mostly involved cough, dyspnea, polypnea and chest pain. Symptoms of ototoxicity mainly included vertigo, in a few subjects tinnitus or hypoacousia. Genital apparatus disorders included female gynaecological symptoms such as vaginal discharge. 


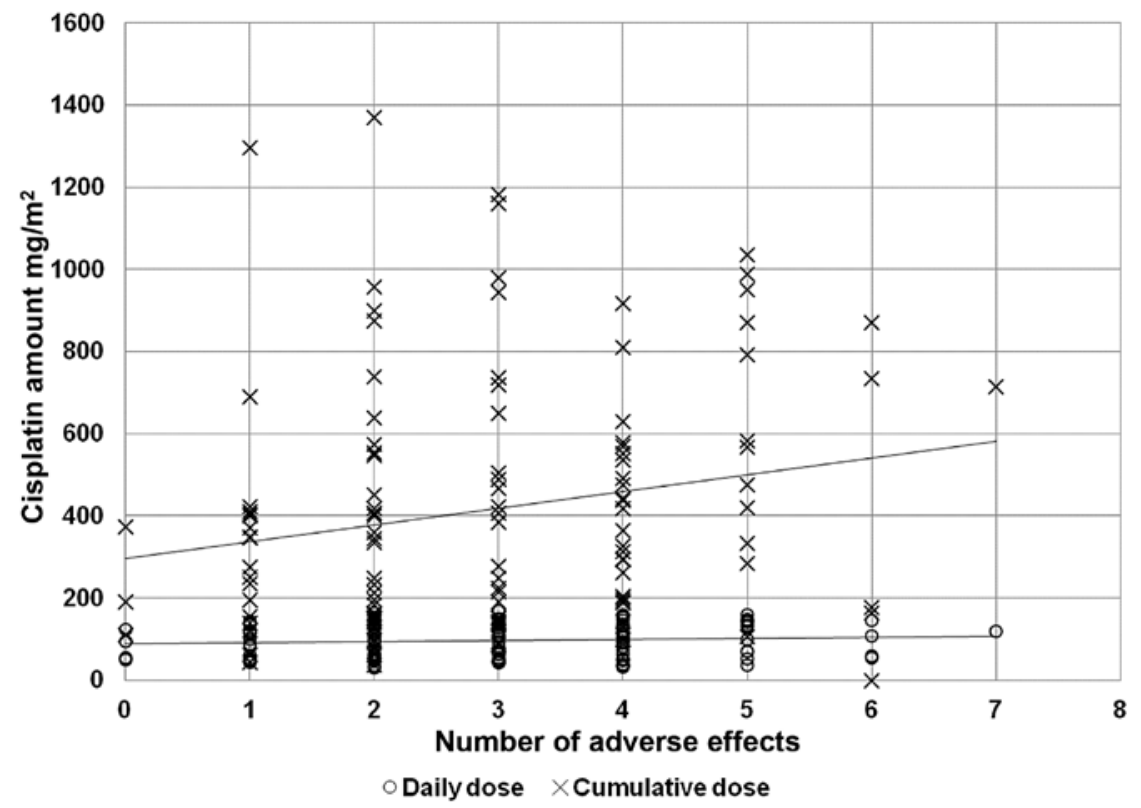

Figure 1. Correlation between administered drug amounts and number of adverse effects. The daily and cumulative cisplatin amounts are expressed in $\mathrm{mg} / \mathrm{m}^{2}$.

Table II. Chemotherapy protocols and adverse effects.

\begin{tabular}{|c|c|c|c|c|c|c|c|c|c|c|c|c|c|c|c|c|}
\hline \multirow[b]{2}{*}{ Adverse effects } & \multicolumn{2}{|c|}{ Cisplatin dose } & \multicolumn{11}{|c|}{ Tumour classes } & \multirow[b]{2}{*}{ Total } & \multirow[b]{2}{*}{$\mathrm{AE}(\%)$} & \multirow[b]{2}{*}{$\mathrm{S}(\%)$} \\
\hline & Day & Cum & $\mathrm{L}$ & GN & $\mathrm{U}$ & GS & $\mathrm{HE}$ & $\mathrm{N}$ & TM & $\mathrm{HN}$ & $\mathrm{M}$ & TY & $\mathrm{O}$ & & & \\
\hline Constitutional symptoms & 0.274 & 0.130 & 47 & 10 & 4 & 2 & 1 & 2 & 3 & 14 & 4 & 0 & 1 & 88 & 23 & 72 \\
\hline Dermatological disorders & 0.500 & 0.860 & 15 & 3 & 2 & 2 & 1 & 2 & 0 & 15 & 3 & 0 & 0 & 43 & 11 & 35 \\
\hline Gastrointestinal disorders & 0.009 & 0.000 & 45 & 14 & 4 & 2 & 1 & 3 & 2 & 13 & 4 & 0 & 1 & 89 & 24 & 72 \\
\hline Genital apparatus disorders & 0.596 & 0.365 & 1 & 0 & 0 & 0 & 0 & 0 & 1 & 0 & 0 & 0 & 0 & 2 & 1 & 2 \\
\hline Haematological toxicity & 0.338 & 0.001 & 29 & 7 & 2 & 2 & 0 & 2 & 3 & 14 & 3 & 2 & 2 & 66 & 18 & 54 \\
\hline Hepatic toxicity & 0.151 & 0.009 & 9 & 2 & 0 & 0 & 0 & 1 & 0 & 1 & 0 & 0 & 0 & 13 & 3 & 11 \\
\hline Nephrotoxicity & 0.264 & 0.950 & 10 & 3 & 1 & 0 & 0 & 1 & 2 & 2 & 1 & 1 & 0 & 21 & 6 & 17 \\
\hline Neurotoxicity & 0.126 & 0.269 & 14 & 3 & 4 & 1 & 0 & 2 & 0 & 4 & 2 & 1 & 1 & 32 & 9 & 26 \\
\hline Ototoxicity & 0.651 & 0.211 & 4 & 2 & 2 & 0 & 0 & 0 & 0 & 3 & 0 & 0 & 0 & 11 & 3 & 9 \\
\hline Respiratory disorders & 0.021 & $\mathbf{0 . 0 3 8}$ & 5 & 2 & 1 & 0 & 0 & 1 & 0 & 1 & 0 & 0 & 0 & 10 & 3 & 8 \\
\hline Sleep-wake disorders & 0.488 & 0.915 & 5 & 0 & 0 & 0 & 0 & 0 & 0 & 0 & 1 & 1 & 0 & 7 & 2 & 6 \\
\hline
\end{tabular}

Spearman non-parametric correlation between daily or cumulative cisplatin dosage and adverse effects, and the number of oncological patients showing the different toxic effects (rows) subgrouped according to the different tumour classes (columns), identified according to the National Cancer Institute Guidelines (2006). Day, cisplatin daily dose; cum, cisplatin cumulative dose; L, lung; GN, gynaecological; U, urothelial; GS, gastric; HE, hepatic; N, neuroendocrine; TM, thymoma; HN, head and neck; M, melanoma; TY, thyroid; O, occult; AE\%, percentage of adverse effects; $\mathrm{S} \%$, percentage of patients affected. Bold print indicates statistical significance; $\mathrm{P}<0.05$ significant, $\mathrm{P}<0.001$ highly significant.

Chemotherapy protocols and adverse effects. Analysis of the average daily doses administered revealed that the most intensive therapy (the highest dosage) was applied for lung sarcoma tumours (treated with $148 \mathrm{mg} / \mathrm{m}^{2}$ of the chemotherapeutic drugs), followed by SCLC (143.75 $\left.\mathrm{mg} / \mathrm{m}^{2}\right)$ and neuroendocrine tumours $\left(136.6 \mathrm{mg} / \mathrm{m}^{2}\right)$. The lowest daily dosage was administered to patients affected by thyroid tumours $\left(45 \mathrm{mg} / \mathrm{m}^{2}\right)$, followed by patients affected by uterine and vulvar tumours $\left(54 \mathrm{mg} / \mathrm{m}^{2}\right)$. The patients receiving a higher CDDP dosage were affected by neuroendocrine tumours (cumulative dose $\left.635.19 \mathrm{mg} / \mathrm{m}^{2}\right)$, followed by those affected by urothelial tumours $\left(621.81 \mathrm{mg} / \mathrm{m}^{2}\right)$ and $\operatorname{SCLC}\left(503.125 \mathrm{mg} / \mathrm{m}^{2}\right)$. The patients receiving the lowest cumulative doses $\left(69 \mathrm{mg} / \mathrm{m}^{2}\right)$ were those affected by ovarian tumours. Finally, doses $<100 \mathrm{mg} / \mathrm{m}^{2}$ were administered to the patients with uterine, gastric, vulvar and thyroid tumours.

In order to verify the correlation between the chemotherapy dosage and the incidence of adverse effects, we plotted the number of toxic effects against cumulative and daily doses of cisplatin administered (Fig. 1), and we correlated these doses 
Table III. Tumour site and adverse effects.

\begin{tabular}{|c|c|c|c|c|}
\hline Adverse effects & Treatment & $\mathrm{SpR}$ & $\mathrm{t}(\mathrm{N}-2)$ & P-value \\
\hline \multicolumn{5}{|l|}{ Gynaecological tumours } \\
\hline Respiratory disorders & EPI & 0.686 & 3.771 & 0.002 \\
\hline Ototoxicity & VP-16 & 0.542 & 2.582 & 0.020 \\
\hline Dermatological disorders & VP-16 & 0.478 & 2.177 & 0.045 \\
\hline Nephrotoxicity & 5-FU & 0.686 & 3.771 & 0.002 \\
\hline Neurotoxicity & Taxol & 0.542 & 2.582 & 0.020 \\
\hline Nephrotoxicity & Taxol & 0.686 & 3.771 & 0.002 \\
\hline Neurotoxicity & Paclitaxel & 0.542 & 2.582 & 0.020 \\
\hline Nephrotoxicity & Paclitaxel & 0.686 & 3.771 & 0.002 \\
\hline Constitutional symptoms & Radiotherapy & -0.620 & -3.162 & 0.006 \\
\hline \multicolumn{5}{|l|}{ Head and neck tumours } \\
\hline Neurotoxicity & Daily dose cisplatin & 0.379 & 2.089 & 0.047 \\
\hline Neurotoxicity & Dexamethasone & 0.556 & 3.407 & 0.002 \\
\hline Constitutional symptoms & Radiotherapy & -0.466 & -2.687 & 0.012 \\
\hline \multicolumn{5}{|l|}{ Lung tumours } \\
\hline Dermatological disorders & Daily dose cisplatin & 0.282 & 2.120 & 0.039 \\
\hline Neurotoxicity & Dexamethasone & 0.312 & 2.368 & 0.022 \\
\hline Nephrotoxicity & Dexamethasone & -0.316 & -2.404 & 0.020 \\
\hline Nephrotoxicity & Radiotherapy & 0.437 & 3.504 & 0.001 \\
\hline Gastrointestinal disorders & Cumulative dose cisplatin & 0.483 & 3.982 & 0.000 \\
\hline Haematological toxicity & Cumulative dose cisplatin & 0.487 & 4.024 & 0.000 \\
\hline Number of adverse effects & Cumulative dose cisplatin & 0.484 & 3.990 & 0.000 \\
\hline \multicolumn{5}{|l|}{ Melanoma } \\
\hline Constitutional symptoms & Daily dose cisplatin & -0.889 & -3.354 & 0.044 \\
\hline Number of adverse effects & Radiotherapy & 0.913 & 3.873 & 0.030 \\
\hline
\end{tabular}

Spearman non-parametric correlation between adverse effects and chemotherapy protocols. SpR, Spearman $\mathrm{R} ; \mathrm{P}$-value $(\mathrm{P}<0.05$ significant, $\mathrm{P}<0.001$ highly significant).

with the associated compound to the adverse effects (Table II). As expected, the cumulative amount of cisplatin was directly related to the number of adverse effects $\left(\mathrm{r}^{2}=0.3826, \mathrm{P}<0.001\right)$. The daily dose correlated with gastrointestinal and respiratory disorders, while the cumulative dose also affected the hepatic and haematological systems.

Tumour site and adverse effects. To study in more detail the side effects detected in the different tumour classes, the number of subjects were determined exhibiting each type of side effect ordered according to tumour class (Table II). No side effect was common to all tumour classes, and the most frequent side effects were gastrointestinal toxicity, constitutional symptoms and haematological toxicity. The constitutional symptoms and gastric toxicity were consistently detected, except in patients with thyroid tumours. Apart from systemic and gastric symptoms, the patient with hepatic tumours also showed dermatological alterations, while patients with lung cancer presented all side effects together. The less common symptoms detected were sleep-wake disorders and alterations of the reproductive, respiratory and auditive tract. The less frequent side effect was genital apparatus toxicity. Following analysis of the number of side effects detected for each tumour class, it was possible to note that the toxicity range was higher in individuals affected by lung cancer, while in those affected by thyroid and hepatic cancer the number of side effects was lower. The effects of chemotherapeutic associations were analysed by Spearman non-parametric correlation. The statistically significant results are reported by tumour and by year of diagnosis in Table III. No correlations were found among chemotherapeutic protocols and adverse effects in thymoma, gastric, occult, neuroendocrine, urothelial, hepatic and thyroid tumours.

Side effects are reciprocally related. Based on the differences in side effects among the tumour classes, we also examined the possible association of side effects together or to any tumour class by Spearman non-parametric correlation. The results, summarised in Table IV, support the hypothesis that dermatological disorders were associated with sleep-wake disorders and haematological toxicity, gastrointestinal disorders were associated with respiratory disorders and ototoxicity, and 
Table IV. Correlation of side effects.

\begin{tabular}{llcrr}
\hline & & $\mathrm{SpR}$ & $\mathrm{t}(\mathrm{N}-2)$ & P-value \\
\hline Sleep-wake disorders & Dermatological disorders & -0.193 & -2.168 & 0.032 \\
Haematological toxicity & Dermatological disorders & 0.236 & 2.676 & 0.008 \\
Respiratory disorders & Gastrointestinal disorders & 0.222 & 2.499 & 0.014 \\
Ototoxicity & Gastrointestinal disorders & 0.184 & 2.058 & 0.042 \\
Ototoxicity & Genital apparatus disorders & 0.197 & 2.210 & 0.029 \\
Hepatic toxicity & Genital apparatus disorders & 0.185 & 2.070 & 0.041 \\
\hline
\end{tabular}

Spearman non-parametric correlation among adverse effects. SpR, Spearman R; P-value ( $<<0.05$ significant, $\mathrm{P}<0.001$ highly significant $)$.

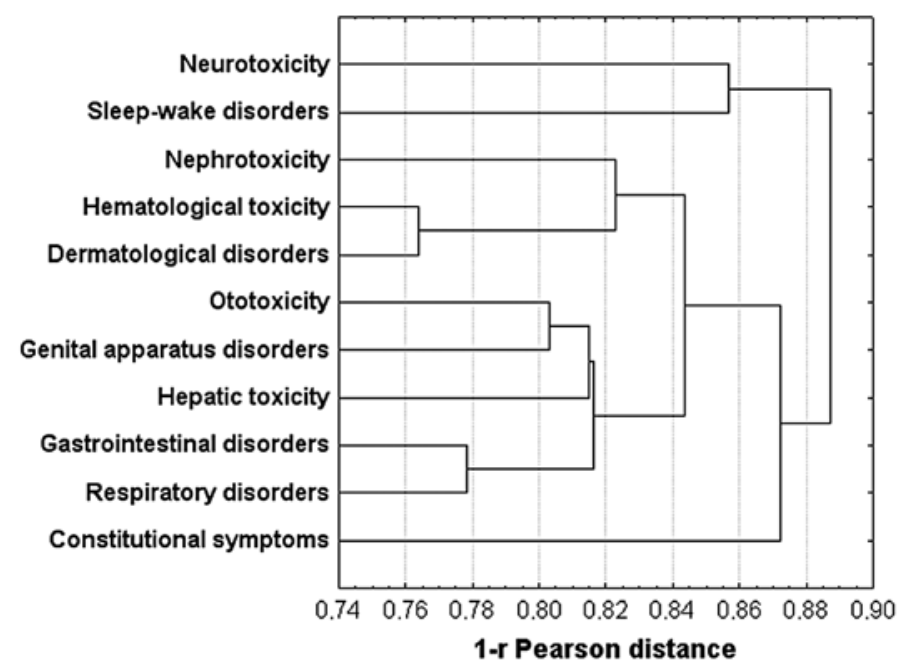

Figure 2. Correlations among side effects calculated by 1-r Pearson distance and plotted according to hierarchical union rank.

genital apparatus disorders were associated with ototoxicity and hepatic toxicity. The last result was also found in the dendrogram of the data plotted using the hierarchical union method (Fig. 2). Notably, neurotoxicity was associated with sleep-wake disorders and, together with constitutional symptoms, stand out from all other groups.

\section{Discussion}

The present study describes the frequency of side effects in 123 patients affected by solid tumours and treated with cisplatin at the St. Anna Hospital of Ferrara, Italy during 2007 and 2008. This is the first study considering heterogeneous populations as previous literature data concern studies limited to specific tumours $(4,39-43)$.

The highest tumour incidence was in males $(63 \%)$, and the most frequent tumour was lung tumour $(43.4 \%$, with a male/female 4:1 ratio and average age of 62 years), as supported by data from Regione Emilia-Romagna (Italy) reported in 2006 (44).

The most frequent adverse effects detected ( $72 \%)$ involved the gastrointestinal apparatus and constitutional symptoms (fever, hyposthenia, asthenia, weight loss and sleep-wake rhythm alterations) while the least frequent effects involved genital apparatus disorders $(2 \%)$. The highest number of side effects was detected in patients with lung cancer; this could be due to the fact that this was the most frequent tumour class. To attenuate the adverse effects of cisplatin treatment, it is necessary to evaluate individual variations due to age, duration of therapy, range of doses and synergism with other compounds causing the toxicity. Considering the therapeutical protocols associated with different tumours, we examined the cisplatin dosages administered. According to this analysis, no direct correlation was noted between the administered daily doses of cisplatin and the other chemotherapeutic drugs involved. During chemotherapy, dexamethasone is applied as an antiemetic (45) and only recently its otoprotective qualities have been recognised in animal models (37). Correlation analyses showed that dexamethasone protects against nephrotoxicity in lung tumour patients. However, a positive correlation between the drug dosage and neurotoxicity was detected in both lung and head-neck tumour patients. In esophageal tumours, the rate of successful cisplatin treatment is $25-35 \%$ for metastatic carcinomas and 50-60\% for local tumours at an advanced stage (18). For head and neck tumours, cisplatin is usually administered in association with 5-fluorouracil and radiotherapy $(7,18)$. In our data set, radiotherapy was inversely correlated to constitutional symptoms, thus, showing a protective effect. In lung tumour patients, the cisplatin dosage was 
the main cause of adverse effects. However, in NSCLC, cisplatin-based chemotherapy shows a complete response only in $\sim 30 \%$ of cases; therefore, it represents only a palliative care treatment (40). Cisplatin is usually administered in association with GEM at the suggested weekly dose of $51 \mathrm{mg} / \mathrm{m}^{2}$ (with gemcitabine $1500 \mathrm{mg} / \mathrm{m}^{2}$ ) (40). In SCLC, cisplatin is usually administered with VP-16; if the tumour is locally advanced, the positive response to chemotherapy is $\sim 50-60 \%$, with an average survival time of 7-11 months. In more advanced tumours, paclitaxel is administered with cisplatin, with a positive response rate of $\sim 34-41 \%$. After chemotherapy, the relapse of tumours occurs in $95 \%$ of cases $(9,31)$. For melanoma treatment, radiotherapy is correlated with the increasing number of adverse effects and decreased cisplatin dosages are associated with constitutional symptoms. The literature data report that cisplatin is administered alone or with VNR and DTIC, at doses $\sim 100-200 \mathrm{mg} / \mathrm{m}^{2}$. The positive response is $\sim 16 \%$ (46). In patients with gynaecological tumours, the cisplatin dosage was not correlated with any adverse effect; in cervical cancer the chemotherapy with cisplatin (not associated with other drugs) usually follows surgical and radiotherapy treatment. Among patients with ovarian cancer, $70 \%$ show an initial positive response to cisplatin but only $<25 \%$ survive up to 5 years. In these tumours, cisplatin is employed in association with paclitaxel or topotecan (3). In the present study group, paclitaxel was correlated with both neurotoxic and nephrotoxic effects. Concerning the other tumour classes, no correlations with adverse effects were detected. In urothelial (or transitional cell) carcinomas, locally advanced or metastatic, and particularly in bladder cancer, cisplatin has been recommended for the last 20 years in association with methotrexate, vinblastine and doxorubicin (M-VAC). This drug combination yielded positive results in $50-70 \%$ of cases, with a $10-20 \%$ complete response and an average survival time of $\sim 1$ year (18). However, the high toxicity of this drug combination promoted successful research on new combinations of compounds, such as gemcitabine, taxane and paclitaxel. Cisplatin combined with gemcitabine and paclitaxel yielded a positive response in $78 \%$ of cases, with an average survival time of 24 month (47). Thymus tumours (including thymoma) at stage III or IV are routinely treated by polychemotherapy mainly involving cisplatin, adriamycin, etoposide, cyclophosphamide or ifosfamide $(48,49)$.

After verifying the most common side effects and which tumour class showed the highest frequency, we also examined the possible associations among the different side effects. The results of these analyses, performed using the Spearman non-parametric correlation test, showed a close relationship between dermatological disorders and either haematological toxicity or sleep-wake disorders, and between respiratory and gastrointestinal disorders, as previously shown in the literature (50).

Other correlations were found between genital apparatus disorders and either ototoxicity or hepatic toxicity, and between ototoxicity and gastrointestinal disorders. These relationships may be due to metabolic effects, but they are difficult to explain as the patient group was highly heterogeneous. Moreover, gastrointestinal disorders are the most common side effects in patients undergoing chemotherapy (50). Ototoxicity is mostly detected in patients with lung or head and neck tumours treated with a high dosage of cisplatin.
In conclusion, through analysis of this heterogeneous group of patients, we confirmed that the main factor influencing the occurrence and severity of adverse effects is the dosage of cisplatin administered, both for single and cumulative doses.

\section{Acknowledgements}

The authors wish to thank all of the personnel working at the Clinical Oncology Unit, St. Anna University Hospital (Ferrara, Italy) for their technical assistance and valuable assistance in collecting the data.

\section{References}

1. Blanchard EM: Cisplatin and solid tumours: still working, after all these years. J Solid Tumors 2: 26-33, 2012.

2. Chaudhary UB and Haldas JR: Long-term complications of chemotherapy for germ cell tumours. Drugs 63: 1565-1577, 2003.

3. Tewari KS and Monk BJ: Gynecologic Oncology Group trials of chemotherapy for metastatic and recurrent cervical cancer. Curr Oncol Rep 7: 419-434, 2005.

4. Armstrong DK, Bookman MA, McGuire W, Bristow RE, Schilder JM and Group GO: A phase I study of paclitaxel, topotecan, cisplatin and filgrastim in patients with newly diagnosed advanced ovarian epithelial malignancies: a Gynecologic Oncology Group study. Gynecol Oncol 105: 667-671, 2007.

5. Ridwelski K, Gebauer T, Fahlke J, et al: Combination chemotherapy with docetaxel and cisplatin for locally advanced and metastatic gastric cancer. Ann Oncol 12: 47-51, 2001.

6. Lee KW, Kim JH, Yun T, et al: Phase II study of low-dose paclitaxel and cisplatin as a second-line therapy after 5-fluorouracil/ platinum chemotherapy in gastric cancer. J Korean Med Sci 22 Suppl: S115-S121, 2007.

7. Cullen KJ, Yang Z, Schumaker L and Guo Z: Mitochondria as a critical target of the chemotheraputic agent cisplatin in head and neck cancer. J Bioenerg Biomembr 39: 43-50, 2007.

8. Vokes EE: Induction chemotherapy for head and neck cancer: recent data. Oncologist 15 (Suppl 3): 3-7, 2010.

9. Niell HB, Herndon JE, Miller AA, et al: Randomized phase III intergroup trial of etoposide and cisplatin with or without paclitaxel and granulocyte colony-stimulating factor in patients with extensive-stage small-cell lung cancer: Cancer and Leukemia Group B Trial 9732. J Clin Oncol 23: 3752-3759, 2005.

10. Bosch ME, Sánchez AJ, Rojas FS and Ojeda CB: Analytical methodologies for the determination of cisplatin. J Pharm Biomed Anal 47: 451-459, 2008.

11. Hohnloser JH, Schierl R, Hasford B and Emmerich B: Cisplatin based chemotherapy in testicular cancer patients: long term platinum excretion and clinical effects. Eur J Med Res 1: 509-514, 1996.

12. Gietema JA, Meinardi MT, Messerschmidt J, et al: Circulating plasma platinum more than 10 years after cisplatin treatment for testicular cancer. Lancet 355: 1075-1076, 2000.

13. Brouwers EE, Huitema AD, Beijnen JH and Schellens JH: Long-term platinum retention after treatment with cisplatin and oxaliplatin. BMC Clin Pharmacol 8: 7, 2008.

14. Tothill P, Klys HS, Matheson LM, McKay K and Smyth JF: The long-term retention of platinum in human tissues following the administration of cisplatin or carboplatin for cancer chemotherapy. Eur J Cancer 28A: 1358-1361, 1992.

15. Gerl A and Schierl R: Urinary excretion of platinum in chemotherapy-treated long-term survivors of testicular cancer. Acta Oncol 39: 519-522, 2000.

16. Gregg RW, Molepo JM, Monpetit VJ, et al: Cisplatin neurotoxicity: the relationship between dosage, time, and platinum concentration in neurologic tissues, and morphologic evidence of toxicity. J Clin Oncol 10: 795-803, 1992.

17. DeConti RC, Toftness BR, Lange RC and Creasey WA: Clinical and pharmacological studies with cis-diamminedichloroplatinum (II). Cancer Res 33: 1310-1315, 1973.

18. Go RS and Adjei AA: Review of the comparative pharmacology and clinical activity of cisplatin and carboplatin. J Clin Oncol 17: 409-422, 1999. 
19. Schweitzer VG: Cisplatin-induced ototoxicity: the effect of pigmentation and inhibitory agents. Laryngoscope 103: 1-52, 1993.

20. Lautermann J, Song B, McLaren J and Schacht J: Diet is a risk factor in cisplatin ototoxicity. Hear Res 88: 47-53, 1995

21. Tanaka F, Whitworth CA and Rybak LP: Influence of $\mathrm{pH}$ on the ototoxicity of cisplatin: a round window application study. Hear Res 177: 21-31, 2003.

22. Bertolaso L, Martini A, Bindini D, et al: Apoptosis in the OC-k3 immortalized cell line treated with different agents. Audiology 40: 327-335, 2001

23. Hyppolito MA, de Oliveira JA and Rossato M: Cisplatin ototoxicity and otoprotection with sodium salicylate. Eur Arch Otorhinolaryngol 263: 798-803, 2006.

24. Previati M, Lanzoni I, Corbacella E, et al: RNA expression induced by cisplatin in an organ of Corti-derived immortalized cell line. Hear Res 196: 8-18, 2004.

25. Arafa HM, Abdel-Hamid MA, El-Khouly AA, Elmazar MM and Osman AM: Enhancement by dexamethasone of the therapeutic benefits of cisplatin via regulation of tumor angiogenesis and cell cycle kinetics in a murine tumor paradigm. Toxicology 222 103-113, 2006.

26. Drottar M, Liberman MC, Ratan RR and Roberson DW: The histone deacetylase inhibitor sodium butyrate protects against cisplatin-induced hearing loss in guinea pigs. Laryngoscope 116: 292-296, 2006

27. Hatzopoulos S, Di Stefano M, Albertin A and Martini A Evaluation of cisplatin ototoxicity in a rat animal model. Ann NY Acad Sci 884: 211-225, 1999.

28. Kartalou M and Essigmann JM: Mechanisms of resistance to cisplatin. Mutat Res 478: 23-43, 2001.

29. Previati M, Lanzoni I, Astolfi L, et al: Cisplatin cytotoxicity in organ of Corti-derived immortalized cells. J Cell Biochem 101: 1185-1197, 2007.

30. Previati M, Lanzoni I, Corbacella E, et al: Cisplatin-induced apoptosis in human promyelocytic leukemia cells. Int J Mol Med 18: 511-516, 2006

31. Rabik CA and Dolan ME: Molecular mechanisms of resistance and toxicity associated with platinating agents. Cancer Treat Rev 33: 9-23, 2007

32. Timmer-Bosscha $\mathrm{H}$, Mulder NH and de Vries EG: Modulation of cis-diamminedichloroplatinum(II) resistance: a review. Br J Cancer 66: 227-238, 1992.

33. Gosland M, Lum B, Schimmelpfennig J, Baker J and Doukas M: Insights into mechanisms of cisplatin resistance and potential for its clinical reversal. Pharmacotherapy 16: 16-39, 1996.

34. Adjei AA, Budihardjo II, Rowinsky EK, et al: Cytotoxic synergy between pyrazoloacridine (NSC 366140) and cisplatin in vitro: inhibition of platinum-DNA adduct removal. Clin Cancer Res 3 : 761-770, 1997.

35. Rybak LP and Whitworth CA: Ototoxicity: therapeutic opportunities. Drug Discov Today 10: 1313-1321, 2005.

36. van den Berg JH, Beijnen JH, Balm AJ and Schellens JH: Future opportunities in preventing cisplatin induced ototoxicity. Cancer Treat Rev 32: 390-397, 2006
37. Daldal A, Odabasi O and Serbetcioglu B: The protective effect of intratympanic dexamethasone on cisplatin-induced ototoxicity in guinea pigs. Otolaryngol Head Neck Surg 137: 747-752, 2007.

38. Astolfi L, Simoni E, Ciorba A and Martini A: In vitro protective effects of Ginkgo biloba against cisplatin toxicity in mouse cell line OCk3. Audiol Med 6: 251-258, 2008.

39. Chen WC, Jackson A, Budnick AS, et al: Sensorineural hearing loss in combined modality treatment of nasopharyngeal carcinoma. Cancer 106: 820-829, 2006

40. Rademaker-Lakhai JM, Crul M, Pluim D, et al: Phase I clinical and pharmacologic study of a 2-weekly administration of cisplatin and gemcitabine in patients with advanced non-small cell lung cancer. Anticancer Drugs 16: 1029-1036, 2005.

41. Rivera F, Vega-Villegas ME, López-Brea MF, et al: Long term results of a phase II trial of induction chemotherapy with uracilftegafur (UFT), vinorelbine and cisplatin (UFTVP) followed by radiotherapy concomitant with UFT and carboplatin (RT/UFTJ) in non-resectable locally advanced (stage IV-B) squamous cell head and neck carcinoma and peripheral blood stem cell support (PBSCS) with febrile neutropenia. Clin Transl Oncol 9: 40-47, 2007.

42. Uhm JE, Lim HY, Kim WS, et al: Paclitaxel with cisplatin as salvage treatment for patients with previously treated advanced transitional cell carcinoma of the urothelial tract. Neoplasia 9: 18-22, 2007.

43. Zuur CL, Simis YJ, Verkaik RS, et al: Hearing loss due to concurrent daily low-dose cisplatin chemoradiation for locally advanced head and neck cancer. Radiother Oncol 89: 38-43, 2008.

44. Regione Emilia-Romagna certified (Internet). Reportistica predefinita REM: anno 2006. (updated 2007 Oct 30). Available from: http://www.regione.emilia-romagna.it/sas/rem/report predef/2006/REM05 2.htm.

45. Kris MG, Hesketh PJ, Somerfield MR, et al: American Society of Clinical Oncology guideline for antiemetics in oncology: update 2006. J Clin Oncol 24: 2932-2947, 2006.

46. Atallah E and Flaherty L: Treatment of metastatic malignant melanoma. Curr Treat Options Oncol 6: 185-193, 2005.

47. von der Maase $\mathrm{H}$ : Current and future perspectives in advanced bladder cancer: is there a new standard? Semin Oncol 29: 3-14 2002.

48. Tiseo M and Ardizzoni A: Chemotherapy in the treatment of thymic tumours. Oncol Rev 2: 95-101, 2008.

49. Girard N, Mornex F, Van Houtte P, Cordier JF and van Schil P: Thymoma: a focus on current therapeutic management. J Thorac Oncol 4: 119-126, 2009.

50. National Cancer Institute: Gastrointestinal Complications $\mathrm{PDQ}^{\circledR}$ 2012, National Cancer Institute, Bethesda, MD (Updated 07/18/2012). Available from: http://cancer.gov/ cancertopics/pdq/supportivecare/gastrointestinalcomplications/ HealthProfessional. 